\title{
Anomalous diffusion in the first-order Jovian resonance
}

\author{
R. R. Cordeiro ${ }^{1}$ and L. A. Mendes de Souza ${ }^{2}$ \\ ${ }^{1}$ Departamento de Física, Universidade Federal de Viçosa, Viçosa - MG, 36571-000, Brazil \\ e-mail: cordeiro@ufv.br \\ 2 Departamento de Física, Universidade Federal de Minas Gerais, Belo Horizonte - MG, 31270-901, Brazil
}

Received 31 January 2005 / Accepted 12 April 2005

\begin{abstract}
A method is presented for the characterization of anomalous diffusive processes in dynamical systems. This method is applied to the analysis of the diffusion in some Hamiltonian systems with special emphasis on the orbital problems. We show that the types of diffusion processes in the borders and in the resonant regions are common for all the studied systems. In the borders the diffusion is governed by an initial exponential stage and in the resonant regions the diffusion is also represented by a power law. In the orbital problem we show that, in general, the resonant asteroids are associated with regions where diffusive processes for the semi-major axis and eccentricity are described by $\sigma \propto t^{H}$, where $\sigma$ is the standard deviation and $\frac{1}{2}<H<1.7$. The values of exponent $H$ were determined in grids of initial conditions in the 2:1, 3:2 and 4:3 Jovian resonances.
\end{abstract}

Key words. diffusion - minor planets asteroids

\section{Introduction}

In Astrophysics, the results of stochastic processes are often used to analyze the dynamics of $n$-body problems, where $n$ is a large number (Chandrasekhar 1941, 1943). However, when modeling the Solar System, it is typical to consider the bodies as weighty particles composing an $n$-body problem where $n$ is not generally a large natural number. When considering the Solar System as an $n$-body problem there is much complexity of motion. In fact, even when considering the System as a three body problem we can find a variety of phenomena that still are not completely explained.

The Solar System's population of small bodies in mean motion resonance has proven to be of research interest and the diffusion process in resonance regions has become an important point of analysis. In a 1996 publication, Varvoglis and Anastasiadis proposed that a power law relating escape time and Lyapunov time can be demonstrated in a connected and finite region, considering the process as Makovian. Murray \& Holman (1997) then determined this relation via a FokkerPlanck equation. Nesvorný \& Ferraz-Mello (1997) used the frequency map proposed by Laskar (1990) and assumed that the variation of frequencies has proprieties of a simple random walk (a random walk with the variance proportional to the time) to estimate the stability of some first order Jovian resonance. Tsiganis et al. (2000) proposed a more general approach to analyze the diffusion process. They considered that the regions of stable chaos (regions where the Lyapunov time is considerably smaller than the stability time: Milani \& Nobili 1997) in phase space are the result of anomalous diffusion: a diffusive process is anomalous if the variance is not proportional to the time.
Other studies have included random processes in their analyses of small body dynamics in the solar system. Considering weak background fluctuating (randomic) forces, Smith (2001) developed a simple model using a linearized equation of motion to analyze the asymmetry observed in the shapes of asteroid families.

A basic question emerges when diffusion processes are considered in studies of dynamic solar systems: what type of diffusion is related to the dynamics of small bodies? In general, the use of the Fokker-Planck equation implies the consideration of random processes with normal diffusion: variance being proportional to time (Uhlenbeck \& Ornstein 1930). As we will argue in the following sections, this type of random process does not correspond to the type of diffusion found when examining orbital problems related with the first-order resonance as well as other Hamiltonian Systems.

This study addresses the type of diffusion found in the firstorder Jovian resonance (diffusion of semi-major axis and diffusion of eccentricity) and in some Hamiltonian systems. For this purpose we introduce a method to determine the anomalous diffusion of ensembles of test particles. This method has as its foundation the calculation of the exponent of the power law describing the evolution of the standard deviation. For the 2:1 and 3:2 resonances with Jupiter, we show that there is a relation between resonant asteroid stability and the region where the diffusion of semi-major axis and eccentricity has the characteristics of an anomalous diffusion process associated with a power law. Our results also provide some arguments corroborating the low population of asteroids in the $4: 3$ resonance.

In the next section, we present a summary of this study's main results regarding the diffusion process. In Sect. 3, we 
show our methodology, using Fractional Brownian motion to show how random processes are related to the orbital problem, and demonstrate that many features of the diffusion process observed in orbital problems are common to other Hamiltonian systems. Section 4 presents our analysis of the relationship between stability and anomalous diffusion in a-e space (semimajor axis versus eccentricity space).

\section{Normal and anomalous diffusion}

Some papers have adopted properties of the random walk model with normal diffusion for their analysis of the connection between stochastic processes and orbital dynamics. A random process exhibits normal diffusion if the respective variance (square of the standard deviation) is proportional to the time: $\sigma^{2} \propto t$. A common example of a stochastic process presenting normal diffusion is the simple random walk, SRW. The one-dimensional SRW is characterized by increments of constant values $\left(\left|\varepsilon_{i}\right|=\varepsilon\right)$ with random directions happening during each time interval $\tau$. If $X(t)$ is the spatial-temporal trace, or path, of the SRW and $\varepsilon_{i}$ is a random variable corresponding to the $i$ th increment, then $\varepsilon_{i}=X(t=i)-X(t=i-1)$ and $X(t=n \tau)=\sum_{i=1}^{n} \varepsilon_{i}$. Furthermore, if the probability of displacement in one direction is the same as displacement in other direction, then the expectation values of $X(t)$ and $X\left(t_{\circ}\right)$ are equal, $\langle X(t)\rangle=\left\langle X\left(t_{\circ}\right)\right\rangle$, and the variance is proportional to the time,

$\sigma^{2}=2 D t$,

where $D$ is the diffusion coefficient. A fundamental point in the determination of the above SRW properties is the use of the ensemble average, $\langle\alpha\rangle$. In fact, the ensemble average is used not so much for the determination of expectation value, $\langle X\rangle$, as it is for the determination of the standard deviation, $\sigma$ (see, for example, Paul \& Baschnagel 1999 or Berg 1983). However, some works based on a single time series employ the time average, $\overline{\alpha(t)}$, (average over a sample) to determine the respective standard deviation; but it is well established that the time average is equivalent to the ensemble average,

$\langle\alpha\rangle=\overline{\alpha(t)}$,

only when the ergodic hypothesis is valid. This well founded supposition should be considered when performing statistical analyzes of diffusion processes in orbital problems.

An immediate extension of the SRW is regular Brownian motion, BM. Usually, in a one-dimensional BM, the displacements follow a Gaussian (normal) probability density of zero mean. Despite the difference in the probability of the distribution of the displacements between the SRW and BM, we also have $\langle X(t)\rangle=\left\langle X\left(t_{\circ}\right)\right\rangle$ and

$\sigma=(2 D t)^{\frac{1}{2}}$

for an ensemble of Brownian particles.

Another property common to SRW and BM is that the trace of both is uncorrelated (each step or increment taken in a SRW or BM does not depend on the history of the trace up to that point). Uncorrelated motions are found in many stochastic processes. The non-correlation of motion is a basic consideration for the deduction of the Fokker-Planck equation (Wang \& Uhlenbeck 1945) and is also a characteristic of Markovian processes (Van Kampen 1982).

Fractional Brownian motion, FBM, is a broader derivative of BM: Mandelbrot \& Van Ness (1968), Mandelbrot (1982) and Feder (1988). The trace for a one-dimensional FBM is defined as

$$
\begin{aligned}
X_{H}(t)= & \frac{1}{\Gamma\left(H+\frac{1}{2}\right)}\left[\int_{-\infty}^{0}\left(\left(t-t^{\prime}\right)^{H-\frac{1}{2}}-(-t)^{H-\frac{1}{2}}\right) \mathrm{d} X\left(t^{\prime}\right)\right. \\
& \left.+\int_{0}^{t}\left(t-t^{\prime}\right)^{H-\frac{1}{2}} \mathrm{~d} X\left(t^{\prime}\right)\right]
\end{aligned}
$$

where $X(t)$ is the continuous (regular and one-dimensional) Brownian motion, $\Gamma$ is the gamma function, and $H$ is a parameter called the Hurst exponent. In the FBM, we also have $\left\langle X_{H}(t)\right\rangle=\left\langle X_{H}\left(t_{\circ}\right)\right\rangle$, with the standard deviation, $\sigma_{H}$, related with the time by

$\sigma_{H}=(2 D t)^{H}$.

After some considerations that do not endanger the generalization, it can also be shown that the auto-correlation function between future increments and past increments is written as (Feder 1988)

$C^{\prime}(t)=\frac{\left\langle-X_{H}(-t) X_{H}(t)\right\rangle}{\left\langle X_{H}(t)^{2}\right\rangle}=2\left(2^{2 H-1}-1\right)$.

Therefore, for $H=\frac{1}{2}$, we have $C^{\prime}(t)$ assuming zero value and BM corresponding to the motion $\left(C^{\prime}(t)=0\right.$ implies an uncorrelated process or Markovian). In fact, as can be observed in Eq. (1), when setting $H=\frac{1}{2}$ we have $X_{1 / 2}(t)=X(t)$ (from Eq. (2) we also have $\sigma_{1 / 2}=\sigma$ ). However, for $H \neq \frac{1}{2}$, we have $C^{\prime} \neq 0$ and there is a correlation between past and future increments. For $\frac{1}{2}<H<1$, we have $C^{\prime}(t)>0$, and the process is correlated and persistent. An increasing (or decreasing) trend of $X_{H}$ in the past implies an increasing (or decreasing) trend of $X_{H}$ in the future. For $0<H<\frac{1}{2}$, we have $C^{\prime}(t)<0$ and the process is correlated and anti-persistent. In this last case, an increasing (or decreasing) trend of $X_{H}$ in the past implies a decreasing (or increasing) trend of $X_{H}$ in the future: Mandelbrot (1982) or Feder (1988).

If the standard deviation of a random walk is related to time by Eq. (2) with $H \neq \frac{1}{2}$, then this process has anomalous diffusive behavior associated with a power law (Metzler et al. 2000). The FBM is not the only random walk that presents anomalous diffusion. Indeed, all diffusive process different from normal diffusion are called anomalous diffusion. So, if the Eq. (2) with $H \neq \frac{1}{2}$ is valid, then the diffusion is anomalous given by a power law.

The Continuous Time Random Walk, CTRW, also presents anomalous diffusion associated with a power law. In the CTRW, the length of a given jump and the waiting time between two successive jumps follow a joint probability density function $\psi(x, t)$ : Metzler et al. (2000). An example of CRTW is the Lévy Walk. In the Lévy walk space and time are delta coupled: $\psi(x, t)=\omega(t) \delta(|x|-v t)$, where $\omega(t)$ is a probability density 

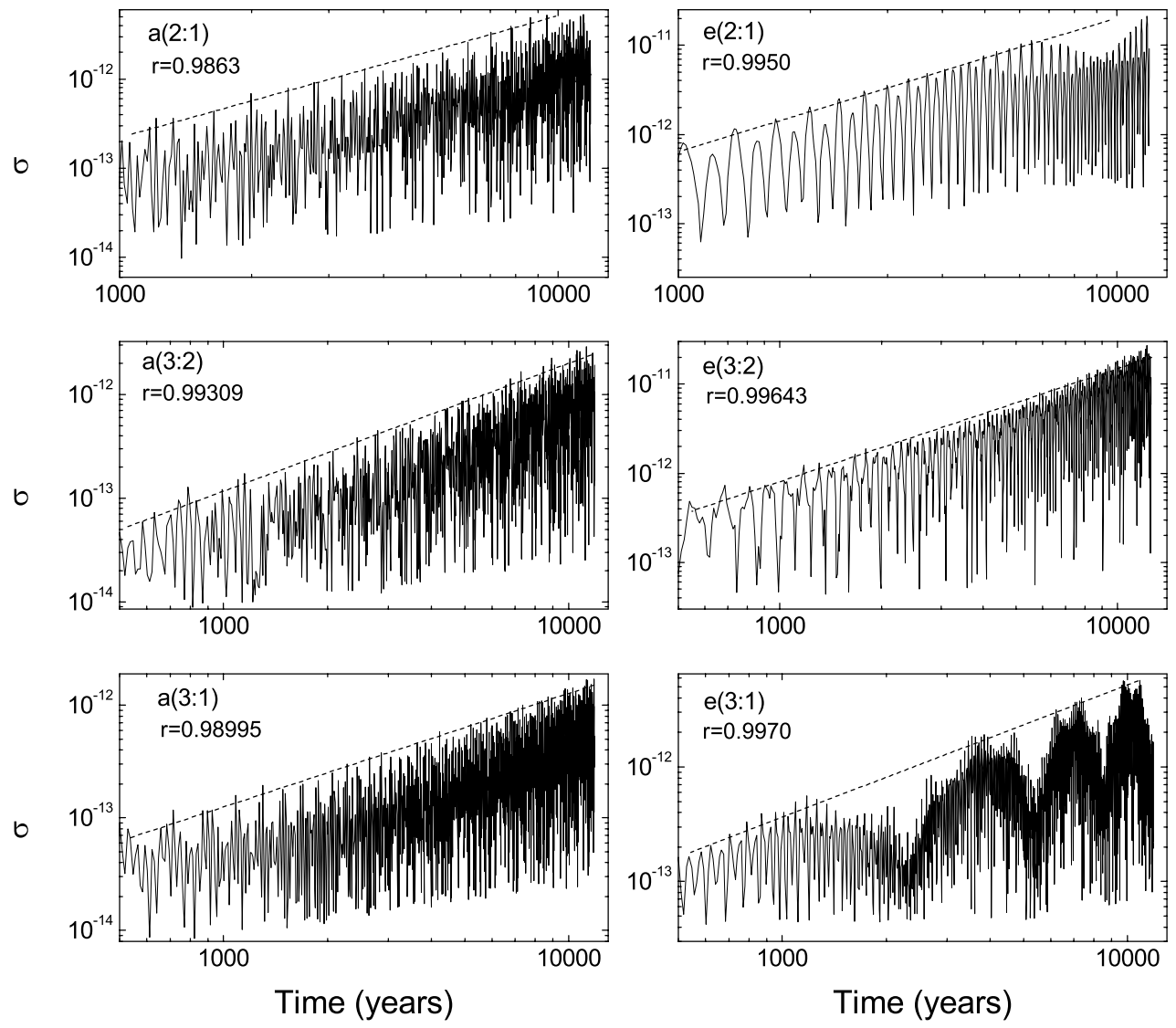

Fig. 1. Evolution of the standard deviation of the semi-major axis and of eccentricity for three ensembles of test particles in the Jovian 2:1, 3:1 and 3:2 resonance regions. The dotted lines correspond to straight lines fitted through the upper points and $r$ is the respective correlation coefficient: see Sect. 3.

function (see Metzler et al. 2000, or Klafter et al. 1996). It is interesting that anomalous diffusion occurs in many Hamiltonian systems and proposals have been made that anomalous diffusion in these systems is a consequence of the transport of chaotic orbits in the region of cantori. This effect is called stickiness: Shlesinger et al. (1993), Zaslavsky (2002), Kandrup et al. (1999) and Efthymiopoulos et al. (1997).

From the equation for $C^{\prime}(t)$ in the second paragraph above, we observe that $H>1$ implies $C^{\prime}>1$, which leads to the FBM always being $H<1$. In fact, it is observed that the amplitudes of the random variations in the trace of a FBM are smaller as $H$ becomes closer to 1 (in the next section we present a procedure to simulate a FBM). Therefore, for $H=1$, the FBM is considered as lacking random characteristics (process with $H=1$ has ballistic diffusion behavior). However, restrictions related to the maximum value of $H$ do not exist for some CTRWs, (Metzler et al. 2000).

\section{Anomalous diffusion in orbital problems}

In general the diffusive processes in the resonant regions of the orbital problems are very complex. In Fig. 1 we show some typical evolutions of the standard deviation for the semi-major axis and eccentricity of three ensembles of 101 massless particles from each of three Jovian resonance regions: 2:1, 3:1 and 3:2 (we also include the 3:1 Jovian resonance although it is a second-order resonance). In each ensemble, we determine the orbits of 101 particles with almost identical initial conditions. These orbits were determined by numerical integration (via Symplect mapping: Cordeiro et al. 1997) of the heliocentric motion equations for the $n$-body problem

$$
\begin{aligned}
& \dot{\boldsymbol{x}}_{i}=\boldsymbol{v}_{i} \\
& \dot{\boldsymbol{v}}_{i}=-k^{2} \frac{m_{\odot}+m_{i}}{\left|\boldsymbol{x}_{i}\right|^{3}} \boldsymbol{x}_{i}+k^{2} \sum_{\substack{j=2 \\
j \neq i}}^{n} m_{j}\left(\frac{\boldsymbol{x}_{j}-\boldsymbol{x}_{i}}{\left|\boldsymbol{x}_{i j}\right|^{3}}-\frac{\boldsymbol{x}_{j}}{\left|\boldsymbol{x}_{j}\right|^{3}}\right)
\end{aligned}
$$

where subscripts $j$ and $i$ are related to $j$-th and $i$-th bodies and the term with the double subscript $j i$ corresponds to the relative position of the $j$-th and $i$-th bodies. The studied system consists of the Sun, Venus, Earth, Mars, Jupiter, Saturn, Uranus, Neptune and particles. The initial conditions were from The Astronomical Almanac for 02/07/1996, and we adopted an integration step corresponding to $1 \%$ of the orbital period of the region (this procedure avoids spurious chaos in resonant regions resulting from the integration, Wisdom \& Holman (1992), and it presents results similar to integrators as the RADAU, Everhart (1985), with a relative error of $10^{-13}$ in situations out of the close approaches). In each ensemble, all particles have the same initial inclinations and longitudes; however, the initial semi-major axes and eccentricities are slightly different. The values of these parameters (for each particle in an ensemble) are give by $a=a_{\circ}+\varepsilon \cos (w)$ and $e=e_{\circ}+\varepsilon \sin (w)$, where $a_{\circ}$ 
and $e_{\circ}$ are the same for all particles, $\varepsilon$ is stochastically and uniformly chosen between 0 and $10^{-13}$, and $w$ is stochastically and uniformly chosen between $0^{\circ}$ and $360^{\circ}$. The inclination, the longitude of perihelion, and the longitude of node were the same as Jupiter's. The mean longitudes for 2:1, 3:2 and 3:1 resonances were chosen so that

$$
\begin{aligned}
& \phi_{2: 1,3: 2}=p \lambda_{\text {Jupiter }}-q \lambda_{\text {particles }}-(p-q) \varpi_{\text {particles }}=0^{\circ} \\
& \phi_{3: 1}=p \lambda_{\text {Jupiter }}-q \lambda_{\text {particles }}-(p-q) \varpi_{\text {particles }}=180^{\circ}
\end{aligned}
$$

where $\phi$ is the critical argument, $\lambda$ is the mean longitude, $\varpi$ is the longitude of perihelion, and $p / q$ corresponds to the commensurabilities of the mean motions: $\approx n_{\text {particles }} / n_{\text {Jupiter }}$ (here we use $\phi$ for the critical argument instead of $\sigma$ considering that in this paper $\sigma$ always corresponds to the standard deviation).

In celestial mechanics, the perturbing potentials can be represented as a sum of harmonic functions of orbital motion longitude. In many cases within the Solar System, the chaotic nature of orbital motion ensures that the longitudes in the perturbing potential behavior as random variables. A very useful and simple model for the understanding of the statistical properties in Fig. 1 consists of a random process associated with a harmonic function,

$$
Y_{H}=\cos \left(w t+X_{H}(t)\right)
$$

where $w$ is a constant and $X_{H}$ is a random variable with anomalous diffusion behavior. To simulate $X_{H}$ we consider it as FBM. In the simulation of FBM, we adopt an approach to Eq. (1) given by

$$
\begin{aligned}
X_{H}= & \frac{1}{\Gamma\left(H+\frac{1}{2}\right)}\left[\sum_{j=1-M}^{i-2}\left((i-j)^{H-\frac{1}{2}}-(i-j-1)^{H-\frac{1}{2}}\right) R(j)\right. \\
& \left.+\sum_{j=i-1}^{i}(i-j)^{H-\frac{1}{2}} R(j)\right]
\end{aligned}
$$

where $R(j)$ corresponds to a Gaussian random increment in $j$-th time and $M$ is the number of steps of the limited memory used in the approximation of the FBM (Feder 1988 or Addison 1997). In Fig. 2 we show three evolutions of standard deviation for $X_{H}$ and $Y_{H}$, considering $H=0.20, H=0.50$ and $H=0.80$ (the standard deviations of phase " $X_{H}(t)$ " and of argument " $w t+X_{H}(t)$ " are equal). In these simulations, we adopted ensembles with 100 particles, $w=1.0 \times 10^{-2}$, $M=5000$ and a Gaussian probability density of zero mean with $\sigma_{\text {Gaussian }}=3.33 \times 10^{-5}$. The dotted lines correspond to the evolution of the standard deviation of $X_{H}, \sigma_{X}(t)$, and the continuous lines correspond to the evolution of the standard deviation of $Y_{H}, \sigma_{y}(t)$. We observe that each dotted line, each $\sigma_{X}$, is the superior limit (envelope) of a continuous line, a $\sigma_{Y}$. Therefore, the envelope's evolution is a strong indicator of the probable evolution of the standard deviation of argument $w t+X_{H}(t)$ (or of the phase $X_{H}$ ) in the harmonic function. Besides, as $X_{H}$ is small $\left(\sigma_{\text {Gaussian }} \approx 10^{-5}\right.$ and $\langle X\rangle=$ $0)$, we can consider that $Y_{H}(t) \approx \cos (w t)-X_{H}(t) \sin (w t)$. So, we regard the expectation value of $Y_{H}$ as the expectation value of $X_{H}$ modulated by functions $\sin (w t)$ and $\cos (w t)$, $\left\langle Y_{H}(t)\right\rangle=\cos (w t)-\sin (w t) \cdot\langle X\rangle$, and the effective evolution of the standard deviation of $Y_{H}$ as similar to the standard deviation of $X_{H}$ with $\sigma_{y}^{2}=\sin ^{2}(w t) \sigma_{X}^{2}$. Moreover, if

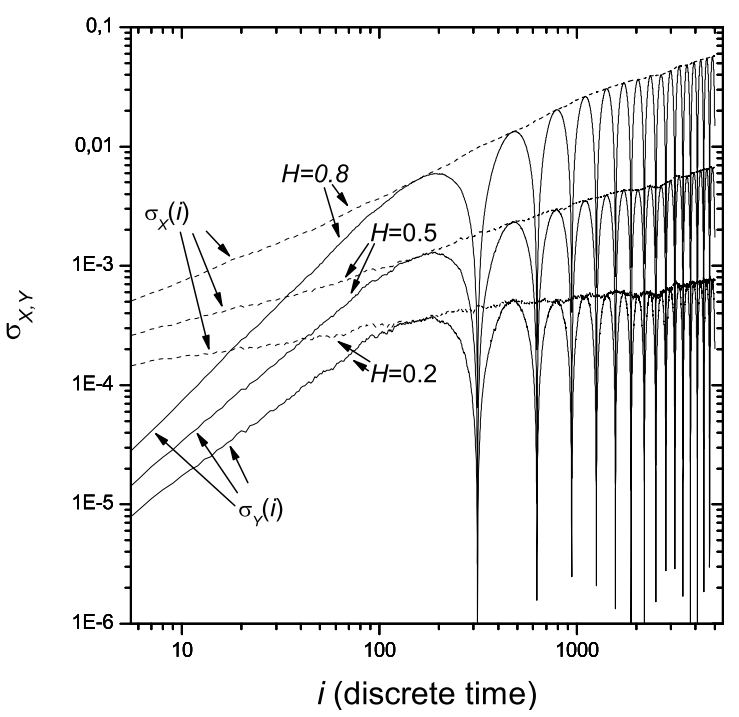

Fig. 2. Evolution of the standard deviation of $Y_{H}$ (continuous lines) and $X_{H}$ (dot lines) for $H=0.20, H=0.50$ and $H=0.80$. We adopt an ensemble with 100 particles and a limited memory corresponding to 5000 steps in these figures.

$Y_{H}(t)=\cos \left(w t+X_{1, H}(t)+\ldots+X_{n, H}(t)\right)$, where $X_{i, H}(t)(i=1, \ldots, n)$ are small independent stochastic variables, then we also have $\sigma_{Y}^{2}=\sin ^{2}(w t) \cdot \sigma_{X}^{2}$ with $\sigma_{X}=\sqrt{\sum \sigma^{2}\left(X_{i, H}(t)\right)}$. Consequently, in following sections we are consider $\sigma_{Y}=\sigma_{X} / \sqrt{2}$ where $1 / \sqrt{2}$ is the effective value of $\left(\sin ^{2}(w t)\right)^{1 / 2}$. Therefore, we consider that if the evolution of $\sigma_{X}$ is a power law as in Eq. (2), then the effective evolution of $\sigma_{Y}$ also is a power law. In this case we refer to the motion of $Y_{H}$ as having an effective anomalous diffusion behavior associated with a power law.

In orbital problems, the evolution of classical elements is not represented by a single harmonic function with a single argument, as in Eq. (4) (analogous with an orbital problem, the argument of Eq. (4), $w t+X_{H}(t)$, could be considered as a mean longitude). As mentioned previously, normally, dynamic equations that represent the evolution of the classical elements are developed as series of harmonic functions, where each harmonic function argument depends on the many terms chaotically evolving. However, it is observed that Fig. 2 is similar to Fig. 1. In Fig. 1 the straight lines through the upper points of the curve for the evolution of the standard deviation approximate the envelopes in the $\log (\sigma) \times \log (t)$ plot in Fig. 2. These upper points were determined by comparing the value of $\log (\sigma(t))$ with the immediately previous and immediately subsequent values: $\log (\sigma(t \pm \Delta t)), \log (\sigma(t \pm 2 \Delta t)), \ldots, \log (\sigma(t \pm n \Delta t))$. In the same plot, we also assumed that an upper point cannot be smaller than the previous upper point. Consequently, instead of calculating the envelopes, we fit a straight line through the upper points of $\log (\sigma(t))$ in all instances (dotted lines in Fig. 1). Then, as previously defined, we can consider the evolution of $a$ and $e$ as demonstrating effective anomalous diffusion behavior associated with a power law in all cases in Fig. 1.

As known, the orbital problem also can present a very complicated structure of phase space with regular motions and chaotic motions. On the borders, in the stochastic layers around unstable manifolds, we observe two stages for the dispersion 

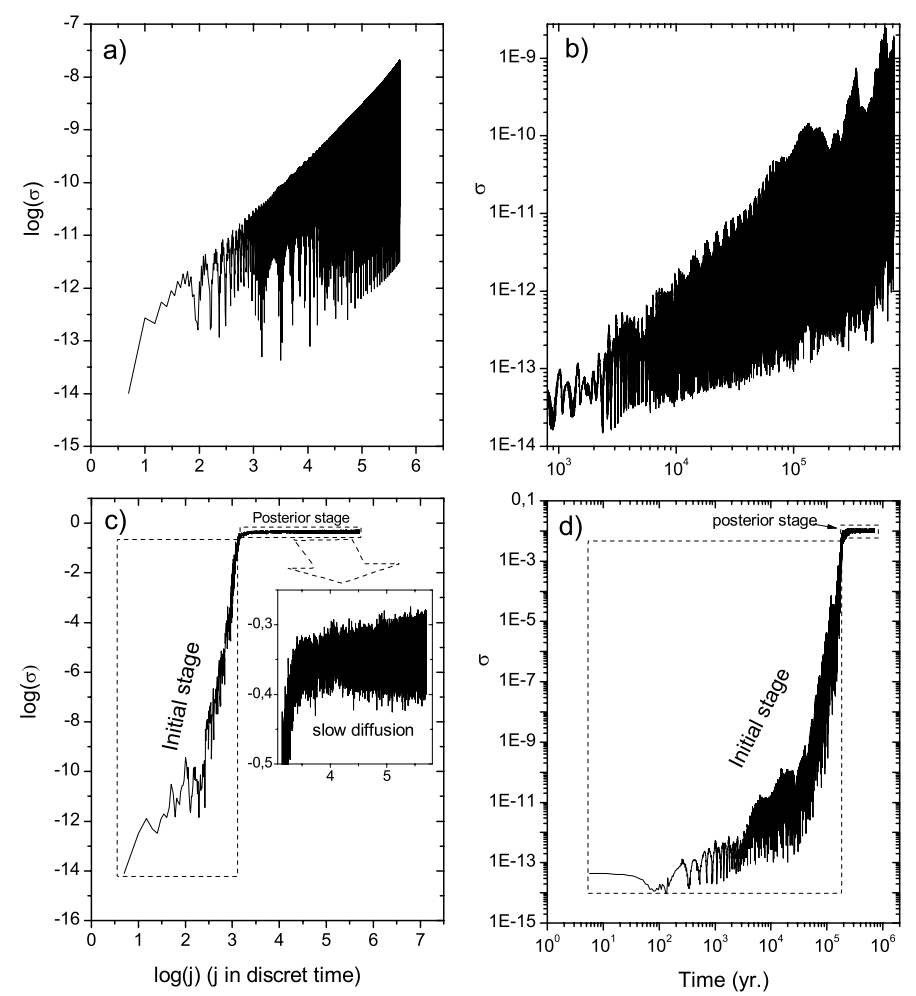

Fig. 3. Evolution of standard deviation of $J$ for the standard map $\left(J_{i+1}=J_{i}+K \sin \left(\theta_{i}\right) ; \theta_{i+1}=\theta_{i}+J_{i+1}\right)$, Figs. 3a,c, and of the semi-major axis for the 2:1 resonance region, Figs. 3b,d. In Fig. 3a we adopt $K=0.9, J_{\circ} \in\left(\pi-10^{-13}, \pi+10^{-13}\right)$ and $\theta_{\circ} \in\left((\pi+0.66)-10^{-13},(\pi+0.66)+10^{-13}\right)$ and in Fig. 3c we adopt $K=0.9, J_{\circ} \in\left(\pi-10^{-13}, \pi+10^{-13}\right)$ and $\theta_{\circ} \in\left((\pi+0.72)-10^{-13},(\pi+0.72)+10^{-13}\right)$. In Figs. 3b and $3 \mathrm{c}$ we adopt the same conditions as described in Sect. 3 with $e=0.3$ and $a=3.1875$ AU for Fig. $3 \mathrm{~d}$ and with $e=0.3$ and $a=3.2375$ for Fig. 3b. Figures 3a and $3 \mathrm{~b}$ correspond to initial conditions in the resonance regions, and Figs. $3 \mathrm{c}$ and $3 \mathrm{~d}$ correspond to initial conditions in the stochastic layers. The small diagram in Fig. $3 \mathrm{c}$ is a magnification of the posterior stage.

process: a fast initial transport along the stochastic layer and a posterior stage corresponding to a slow diffusion process. In the initial stage along the stochastic layer, we observe an exponential relation between the standard deviation and the time,

$\sigma=a \mathrm{e}^{b t^{c}}$

with $a, b$ and $c$ being constants. In general the exponential relation between $\sigma$ and the time is not a characteristic of erratic and disordered motion. For example, this process also can happen in symplectic linear maps. We therefore refer to the initial stage process as dispersion and not as diffusion. The initial stage continues until reaches a threshold, when the ensemble is completely dispersed in the stochastic layer. After the threshold is passed, the dispersion process is considerably reduced, only presenting a "slow diffusion". In this later stage, due to the dispersal of the ensemble, slow diffusion becomes more a property of the stochastic layer than of the region of the initial condition. These stages also can be observed in very simple systems as the standard map. For the standard map, we observed that all ensembles with initial conditions in the same stochastic layer present the former stages, independent of the stochastic layer's widths. Transverse dispersions on the stochastic layer also occur. These processes are usually very fast and are only observed during the first instant. If the stochastic layer is narrow, then the values of transverse standard deviation are proportionately limited.
Out of the borders, in the resonant regions, we note other processes. In general, we observe diffusion processes in resonant regions that correspond to a power law, as Eq. (2). These facts are illustrated in Figs. 3a, and 3b for the standard map and for the orbital problem in the $2: 1$ region.

In the center of the resonant region, on the stable stationary solutions, the evolution of standard deviation does not show diffusion. The evolution of the standard deviation only shows a limited oscillation with values smaller than $10^{-12}$. However, for the initial conditions of the ensemble near to but out of the center of the resonant region, we observe an initial stage without evolution for the standard deviation and also a posterior stage of anomalous diffusion following a power law. The life-time of the initial stage without evolution for the standard deviation is shorter as the initial conditions are more distant from the center of the resonance. This phenomenon was observed in orbital problems and in the standard map. Because of this, we also introduced an extra condition for the determination of the upper points in the plots $\log (\sigma) \times \log (t)$ : a point is an upper point if also $\sigma>10^{-12}$.

In Hamiltonian systems, the power laws, as in Eq. (2) with $H \neq \frac{1}{2}$, are typical of "stranger" or "fractional" kinetics. Theoretical works (see Zaslavsky 2002) suggest that the power law is the consequence of a phenomenon known as stickiness, which consists of the transport of the orbits through the gaps of Cantorus. This kinetic has 
characteristics similar to the Levy processes that have been adopted as an alternative to the Gaussian processes (see Shlesinger et al. 1993; Zaslavsky 2002). We never observe anomalous diffusion associated with power laws in stochastic layers through the method of ensembles. On the contrary, from our investigations the power law only appears for ensembles in resonant regions. However the resonant regions have many narrow islands and stochastic layers that are even narrower. Exponential decays and power laws are observed in many other phenomena of conservative dynamical systems. For example, Kandrup et al. (1999) showed that for some autonomous twodegrees-of-freedom Hamiltonian systems, the escape of orbits can present evolution for the escape probability associated with exponential decay and power law decay. They argue that these phenomena are associated with the break of KAM tori and with the stickiness (see also Efthymiopoulos et al. 1997 for a very good investigation of the phenomenon of the stickiness in the standard map).

The time of integration adopted for the orbital problems in the next section is smaller than the one needed to observe all processes occurring at the borders. However, as we show in the next section, the slope of the exponential relation between $\sigma$ and the time for the initial stage in the stochastic layers is greater than the slope of the power law, Eq. (2), in the resonant regions (in the plot $\log (\sigma) \times \log (t)$ the slope of the power law corresponds to the exponent $H$ ). Therefore, even a small interval of integration can be used to identify and distinguish the border and resonant regions using the proposed method. In general, the borders are associated with $H \geq 1.7$. The adopted integration time for the orbital problems in the next section is smaller than the typical secular period for $a$ and $e$ in the studied resonant regions. However, the diffusion processes are determined by the use of ensembles of test particles with almost identical initial conditions. So, the main changes undergone by a test particle also happen to other particles of the same ensemble. This fact permits us to identify the kind of diffusive process without the need to filter out the almost periodic changes of the orbital elements. We present in Figs. 3a,b two examples of evolution of the $\log (\sigma)$ in resonant regions with time intervals larger than the main periods of change of the respective parameters. In these figures we observe that the evolution of the upper points does not present a periodic change but rather a monotonous evolution. So, we consider the above procedure as valid to characterize the diffusion in the studied regions. We used small times of integration to reduce computation time and because they provide other benefits to be further discussed in the next section. It is not often that processes are as well defined as the ones in Fig. 3. Often situations appear in which different order resonances overlap, which leads to a more vague definition of the type of diffusion process in action.

\section{Diffusion in resonance regions}

The determination of the standard deviation's evolution for the semi-major axis, $\sigma_{a}$, and eccentricity, $\sigma_{e}$, by use of an ensemble of particles, as in Figs. 1-3, can be applied advantageously to phase space regions where the processes are ergodic as well as to regions where the ergodic hypothesis is not valid. In addition, this method is also useful when solving for orbital problems as in Fig. 1 and Figs. 3b,d: the type of diffusion process in a region of $a-e$ space can be determined for small intervals of integration of the Eq. (3) (each integration finishes after the determination of 40 upper points, at least, for $\sigma_{a}$ and $\sigma_{e}$, with $\sigma_{a}, \sigma_{e}>10^{-12}$ for the upper points, or up to the time of integration to reach a limit value). Even for ergodic regions, the determination of standard deviation variation as a function of time by integrating a single particle using the time average generally requires a long integration interval, but a question emerges in this case: is this variation of the standard deviation representative of the region of the initial condition? The answer depends on the value of exponent $H$ associated with the ensemble's initial conditions and the integration interval. For $H>1 / 2$ (for superdiffuse behavior), and for long integration times, an orbit can move away from the initial region and thereby disable the characterization of the diffusion process.

In our studies of diffusion processes in resonance regions, we adopted the same model and a procedure similar as that used to develop Fig. 1 (described in the previous section) and Figs. 3b,d. A grid is used to divide the $a-e$ space of each region around a resonance and to each box of the grid we associate an ensemble. We then determine the value of the orbital parameters in each step of the integration and for each particle of the ensemble. These calculations lead to a value for standard deviation, and, after a number of steps, an understanding of its evolution.

For each box in $a-e$ space, we fit a straight line through the upper points of the oscillations of $\log (\sigma(t))$ and compute the correlation coefficient for this linear fit. As mentioned in previous sections, the effective exponent $H$ corresponds to the slope of the straight line through the upper points.

\subsection{The 2:1 resonance}

Figure 4 shows maps of the effective exponents $H$ (Figs. 4a,c) and of the correlation coefficients (Figs. 4b,d) for the fit of a straight line across the upper points of the 2:1 Jovian resonance region considering the diffusion of the semi-major axis (Figs. 4a,b) and the eccentricity (Figs. 4c,d). In the effective exponent $H$ maps (EH-maps), the gray areas correspond to initial conditions in which $0<H<1.7$, the hatched areas correspond to initial conditions with $H=0$, and the blank areas correspond to initial conditions where $H>1.7$ and the diffusive transport in $a-e$ space occurs more rapidly. In the correlation coefficient maps (CC-map), the blank areas correspond to the region where the upper points present a small dispersion around the fitted straight lines.

In Fig. 4a, we observe the borders regions and the location of the center of the 2:1 resonance (see Nesvorný \& Ferraz-Mello 1997 for the location of the border and of the center of the 2:1 resonance). The borders are shown as a portion of the blank area in the form of a V and a part of the location of the resonance center crosses the hatched central area vertically. The dispersion of $a$ and $e$ at the border is more rapid than in other areas. As we previously mentioned, the diffusion at the borders is not represented by a power law but with an 

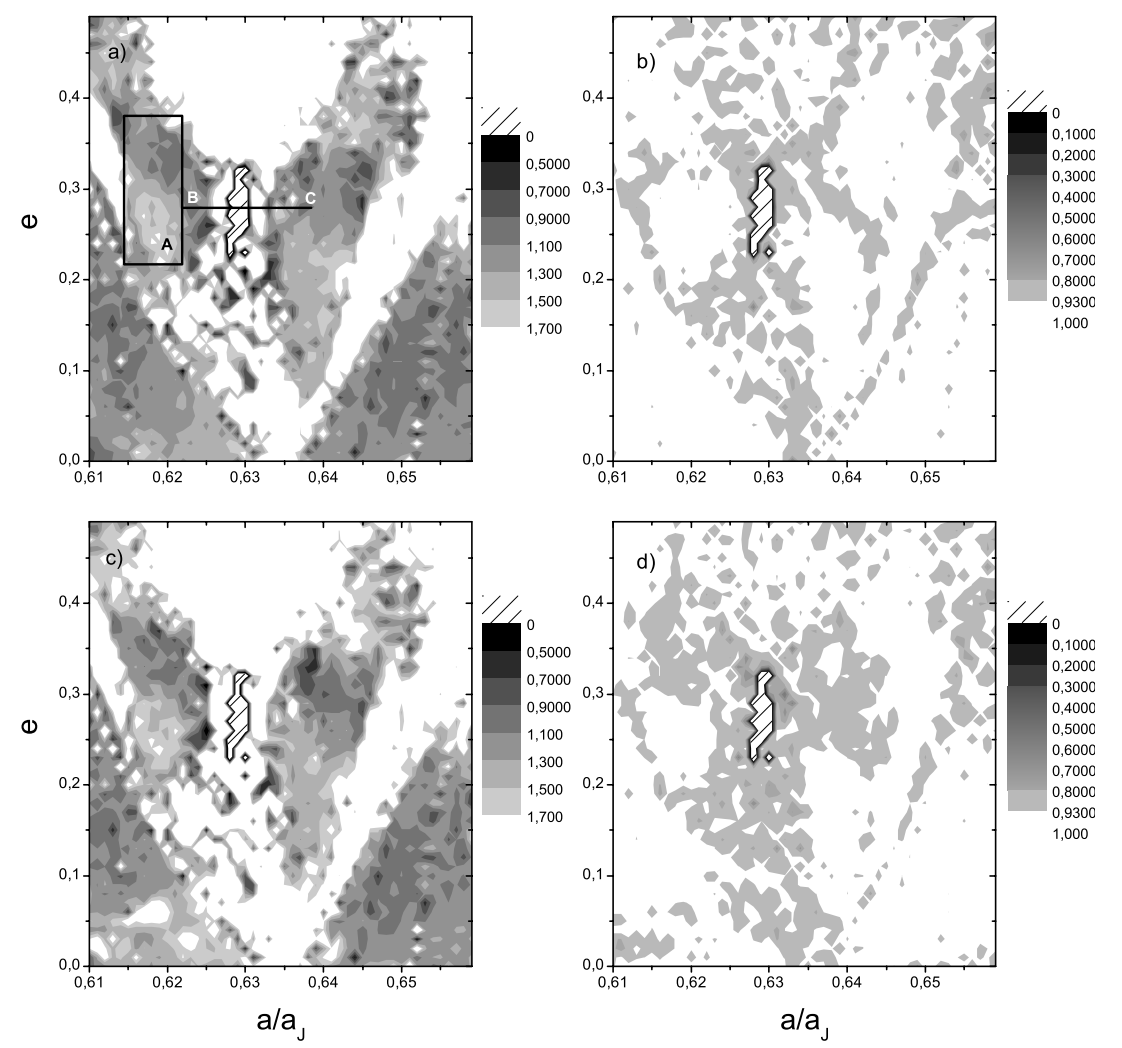

Fig. 4. Exponent effective $H(\mathbf{a}, \mathbf{c})$ and correlation coefficient maps $(\mathbf{b}, \mathbf{d})$ for the $2: 1$ resonance region. The correlation coefficient corresponds to the fit of the straight line across the upper points of $\log (\sigma) \times \log (t)$. Figures $4 \mathrm{a}, \mathrm{b}$ correspond to diffusion of the semi-major axis and Figs. $4 \mathrm{c}, \mathrm{d}$ correspond to eccentricity. The rectangle A corresponds to the region of the proper elements of resonant asteroids computed by Roig et al. (2002), and the segment B-C corresponds to the initial conditions of the analysis of the first instant when the standard deviations are greater than $10^{-13}$ (see Fig. 6).

exponential law. This fact is corroborated by the CC-maps, Figs. 4b,d, where we also observe that at the borders the correlation coefficients for the fit of the power law are smaller.

In the hatched central area, the region where a part of the resonance center is located, the diffusion is slow, and for the adopted interval of integration $\sigma_{a}$ is always less than $10^{-12}$ (for the $2: 1$ resonance we adopt a limiting value for the integration equal to 4800 periods of Jupiter). In our calculation, we only began to compute the upper points after the first instant when $\sigma_{a}$ and $\sigma_{e}$ are both greater than $10^{-12}$. Up to 3000 years, we do not observe anomalous diffusion following a power law for initial conditions in the hatched area. After 3000 years, anomalous diffusion associated with a power law is observed. In general, in the hatched area we have $\sigma<10^{-12}$ for a time of integration smaller than 57120 years $(\approx 4800$ periods of Jupiter).

In Fig. 5, we show the typical evolution of $\sigma_{a, e}$ for an ensemble of particles in the hatched area. We observe that, although $\sigma_{e}$ begins to be bigger than $10^{-12}$ for $t \cong 4000$ years, $\sigma_{a}$ continues to be less than $10^{-12}$ for all the adopted integration times, as shown in Fig. 5.

In Sect. 3, we noted that the evolution of $\sigma_{a, e}$ close to the center of the resonant region shows two stages: an initial stage without evolution of $\sigma_{a, e}$ and a later stage of anomalous diffusion following a power law. Figure 6 shows the first instant when $\sigma_{a}>10^{-13}$ for 35 ensembles in segment B-C of Fig. 4a.

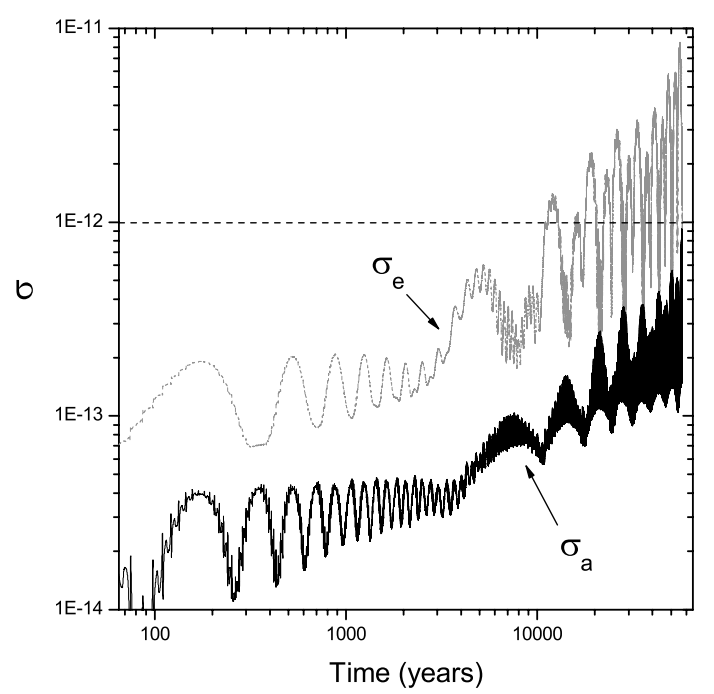

Fig. 5. Evolution of $\sigma_{a}$ and $\sigma_{e}$ for an ensemble of particles with initial conditions around $e=0.295$ and $a=3.26965 \mathrm{AU}$.

Although we have adopted for the calculation of $H$ the first instant when both $\sigma_{a}>10^{-12}$ and $\sigma_{e}>10^{-12}$, for the beginning of the recording of $\sigma_{a, e}$, we also observed that, in the hatched area, $\sigma_{a}<10^{-13}$ during in the initial stage without evolution of $\sigma_{a}$ as a power law. Therefore, in the hatched central area, $\sigma_{a}>10^{-13}$ corresponds to anomalous diffusion following a 


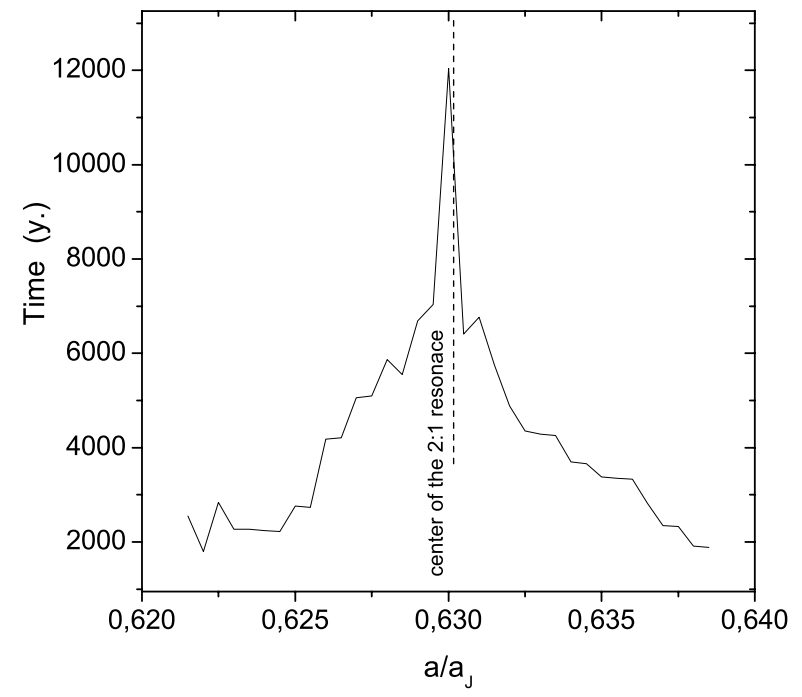

Fig. 6. The first instant when the standard deviations of semi major axis and eccentricity are greater than $10^{-13}$ for ensembles with initial conditions in the segment B-C of Fig. 4a.

power law, and from Fig. 6 we conclude that the closer the ensemble is to the center of the resonance the greater the integration time needed until the power law is first observed, substantiating the statement made in Sect. 3. We note that the location of the center of the 2:1 Jovian resonance is not a typical region of quasi-regular motion. Using frequency map analysis, Nesvorný \& Ferraz-Mello (1997) showed that there are chaotic orbits at the location of the 2:1 resonance, although for $e \approx 0.3$ there are some regular orbits.

From the qualitative point of view, the EH-map for eccentricity exhibits the same characteristics as the EH-map for the semi-major axis. The CC-maps, Figs. 4b,d, show a great number of ensembles in the resonance region that present considerable dispersion of the upper points around the fitted straight line.

The dynamic and size distribution of asteroids in the 2:1 resonance was studied by Roig et al. (2002). In this analysis, Roig et al. (2002) adopt a definition of proper elements $\left(a_{p}, e_{p}\right)$ similar of that of Morbidelli et al. (1995): the values of $a_{p}$ and $e_{p}$ correspond to the average values of $a$ and $e$ when

$\phi=0 \quad$ and $\quad \varpi-\varpi_{J}=0$,

where subscript $J$ refers to Jupiter. Roig et al. (2002) determined the approximate values of $a_{p}$ and $e_{p}$ for each asteroid in the 2:1 Jovian resonance (these average values are referred to as pseudo-proper elements). They integrated the orbits of 53 numbered and unnumbered multi-positional asteroids for $0.2 \mathrm{Myr}$, determining the approximate values of $a_{p}$ and $e_{p}$ and their respective standard deviations. The values of the elements were registered when simultaneously $|\phi| \leq 5^{\circ},\left|\varpi-\varpi_{J}\right| \leq 5^{\circ}$ and $\dot{\phi}>0$, after removing the short-period variations related to Jupiter with a digital filter. The relations established in (5) for the determination of $a_{p}$ and $e_{p}$ are in agreement with the initial conditions adopted in the EH-map and the CC-map; however, we also consider $\Omega-\Omega_{J}=0$ in our initial conditions $(\Omega$ is the longitude of the ascending node). We observe in Fig. 2 of
Roig et al. that most pseudo-proper elements of the 51 resonant asteroids are in rectangle $\mathrm{A}$, a region with anomalous diffusion behavior and $H<1.7$.

Our more stable regions, with slower diffusion, correspond to the most stable regions found by Nervorný \& Ferraz-Mello (1997). Klafke (2001) also studied chaotic diffusion through a map of standard deviations for the 2:1 resonant region. He also adopted a grid for initial conditions and considered only one initial condition per box. In each box he determined the standard deviation of the maximal values for semi-major axis, eccentricity and inclination: the maximum values of the classical elements were determined in intervals of time. For the 2:1 resonance region, Klafke showed that the center of the resonance does not correspond to the more stable region (a region with small standard deviations). This is more evident in Klafke's map for eccentricity, which shows that the more stable regions (small values of $\sigma_{e}$ ) are above the borders, corresponding to our regions of anomalous diffusion associated with the power law.

It was significant that the most stable resonant asteroids exhibit diffusion processes related to anomalous diffusion with $H<1.7$. As we will show in the next section, this fact is more evident in the $3: 2$ resonance.

\subsection{The 3:2 resonance}

In Fig. 7, we show the EH-maps and CC-maps for semi-major axis and eccentricity in the 3:2 Jovian resonance region. As in Fig. 4, the gray scale on the EH-maps (Figs. 7a,c) corresponds to $H<1.7$, and the blank areas on the CC-maps (Figs. 7b,d) correspond to the values of the correlation coefficients of the envelope close to one. The gray region in Fig. 7a, corresponding to the anomalous diffusion of the semi-major axis associated with the power law with $H<1.7$, is denser than that found in Fig. 4a (the 2:1 resonance). A similar relationship between Fig. 7c, anomalous diffusion of the eccentricity, and Fig. 4c is not obvious. We also observe in Fig. 7a that, in general, the center region with $H<1.7$ has larger $H$ values than the sides. This implies that diffusion of the semi-major axis is faster in the center of the gray region than in its sides. For the diffusion of the eccentricity, we observe a dark central area associated with $H<0.5$ adjacent to a clear central area associated with $H>1.7$. The dark central area is associated with small correlation coefficient values while the adjacent clear area is associated with correlation coefficient values that are close to 1 . From Figs. 7b,d, we observe that the number of ensembles with correlation coefficients close to 1 is larger for the semi-major axis than for eccentricity. However, even for eccentricity, we found that the CC-maps for the 3:2 resonance have more ensembles with high correlation coefficients than the $2: 1$ resonance. Nesvorný \& Ferraz-Mello (1997) found that the more regular orbits are found in the center of the resonant region and that orbits are more chaotic when the initial conditions are more distant from the center of the resonance. They also found that outside of the gray region, where $H>1.7$, the orbits are highly chaotic.

The hatched areas with $a / a_{J}<0.75$ in Fig. 7a correspond to the initial conditions presenting higher instability while the 

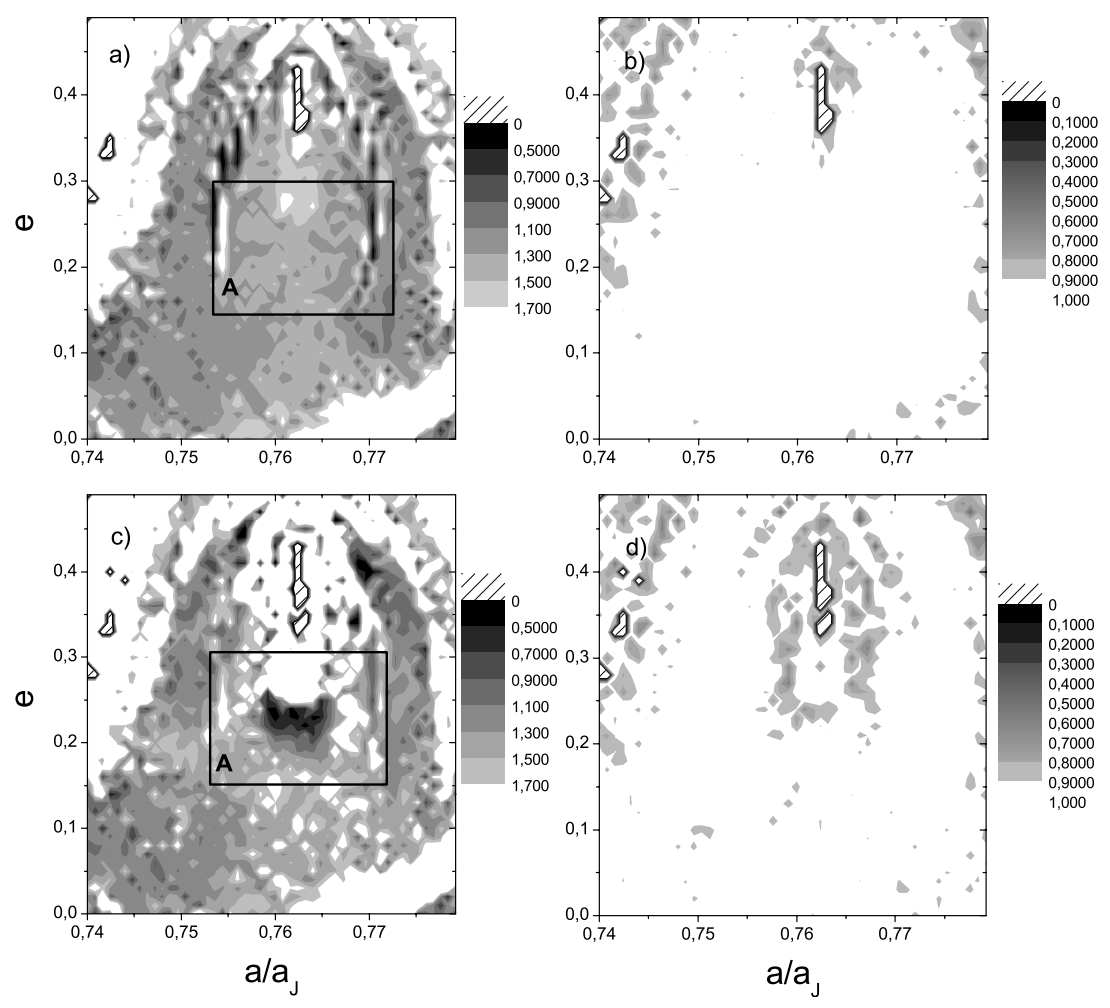

Fig. 7. Same as Fig. 4 for the 3:2 resonance.

other hatched central area corresponds to the ensembles presenting slow anomalous diffusion. The hatched central area is characterized by processes similar to those observed in the hatched area of Fig. 4a: $\sigma_{a}$ is smaller than $10^{-12}$ for all intervals of integration adopted. For the 3:2 resonance, we use an integration time equal to 6211 periods of Jupiter.

Comparing Nesvorný and Ferraz-Mello's (1997) Fig. 1 with our region of anomalous diffusion behaviors with $H<$ 0.17 , we observe that for most of the detected resonant asteroids in the 3:2 resonance, $a_{p}$ and $e_{p}$ are in the region delimited by rectangle $\mathrm{A}$. The values of $a_{p}$ and $e_{p}$ in Nesvorný and Ferraz-Mello's Fig. 1 correspond as much to $\dot{\phi}>0$ as $\dot{\phi}<0$. It has been ascertained that for $\dot{\phi}<0$, we have $a_{p}<a_{\text {res }}$ ( $a_{\text {res }}$ corresponds to location of resonance) and for $\dot{\phi}>0$, we have $a_{p}>a_{\text {res }}$. Also, we observe that in Nesvorný and FerrazMello's' Fig. 1, with the exception of two bodies, the resonant and asteroids of the Hilda group are below the planetary crossing line.

\subsection{The 4:3 resonance}

In Fig. 8, we show the EH-maps and CC-maps for the 4:3 Jovian resonance region. Results for the semi-major axis are depicted in Figs. 8a,b and results for eccentricity are depicted in Figs. 8c,d. The left limit of the gray area in Figs. 8a,c is associated with a border of the 3:2 resonance (see the S2 line in the Nesvorný and Ferraz-Mello's Fig. 1). The right limit of the gray area in Figs. 8a,c is probably associated with a border of the 5:4 resonance. In fact, the intersection of the $4: 3$ resonance with the $3: 2$ and 5:4 resonances restricts the area of the 4:3 resonance of more regular orbits.
The hatched areas in Figs. 8b,d are ensembles with large numbers of orbits that undergo close approaches to Jupiter. As in the 3:2 resonance region, in the EH-map for eccentricity we have a dark central region with $H<0.5$ adjacent to a clear central area with $H>1.7$. Again the dark area is associated with low values of the correlation coefficient and the adjacent clear area is associated with values of the correlation coefficient close to 1 . Here we do not observe a hatched central area associated with the slow diffusion. We also observe that Thule (the blank cross in Fig. 8c) is on the gray area with $H<1.7$.

The intervals of time without occurrence of a planetary crossing are short in the 4:3 resonance region due to change in the orbital elements. In Fig. 9, we show initial conditions in the $3: 2$ and $4: 3$ resonant regions that lead to planetary crossings or closest approaches taking up to 378 years. We again divide the $a-e$ space into a grid and consider the center of each box as an initial condition (considering $\phi=0$ and $\varpi-\varpi_{J}=0$ ). As shown in Fig. 9, the quasi-totality of initial conditions that result in planetary crossings or close approaches taking place within 378 years occurs within 10 years. In the 4:3 region, we find a small connected area of initial conditions (hatched area) where planetary crossings and closest approaches take more than 378 years. We also observe that Thule (the white circle) is found on the border of this area.

\section{Conclusions}

As we have shown in previous sections, a random walk with anomalous diffusion seems to be a more adequate model to describe the diffusive processes in regions of first-order Jovian resonance than a random walk with normal diffusion. From the 


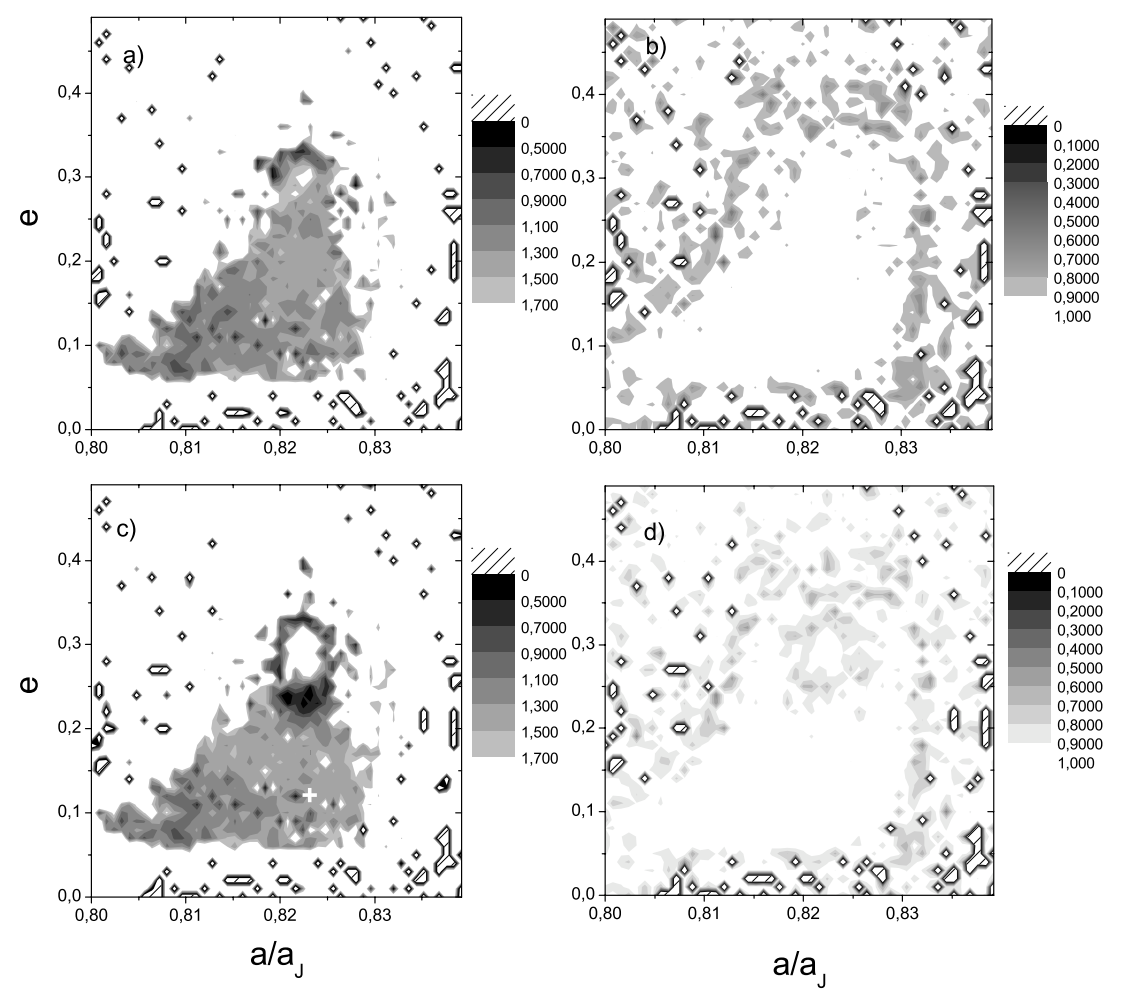

Fig. 8. Same as Fig. 4 for the 4:3 resonance. The cross corresponds to the proper elements of Thule.
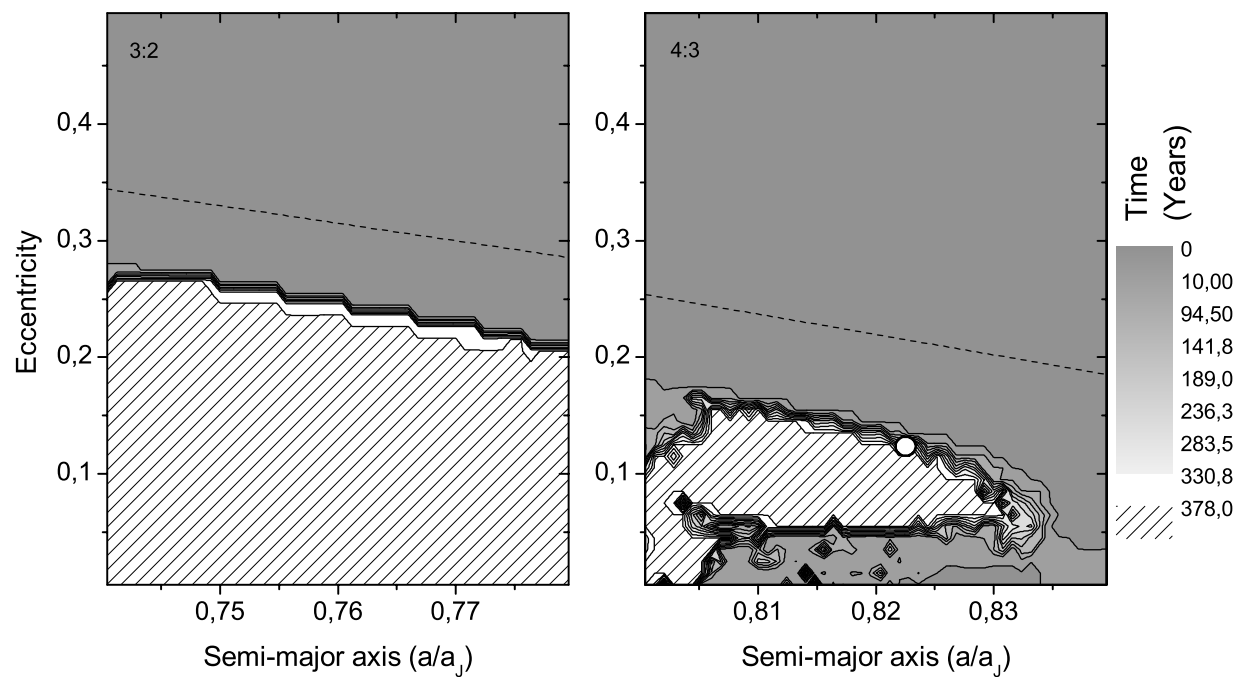

Fig. 9. Initial conditions in the 3:2 and 4:3 resonant regions where the planetary crossings and closest approaches take up to 378 years. The hatched area corresponds to the initial conditions where the planetary crossing and the closest approach take longer than 378 years and the white circle in diagram 4:3 corresponds to Thule. The dotted lines correspond to planetary crossings of Jupiter.

calculation of exponent $H$ via the ensembles of the test particles, we have a reliable portrait of the regions where the process of anomalous diffusion processes can occur. These processes are common to many Hamiltonian systems:

- in the resonance borders the diffusion is initially manifested as an exponential law and later it is manifested as slow diffusion almost without evolution of standard deviations;

- in the resonant region, the standard deviation does not initially show the occurrence of diffusion. After a time interval, the diffusion acts as anomalous diffusion associated with a power law. The closer the initial conditions are to the center of the resonant region the longer the interval of time of the first phase.

The regions of first-order mean motion Jovian resonance show that the observation of a resonant asteroid is more likely in regions of anomalous diffusion for the semi-major axis and eccentricity: regions where the respective standard deviations are related to time by a power law with $0.5<H<1$.7. We found a small area of initial conditions in the $4: 3$ resonance that lead to planetary crossings taking more than 378 years. This small 
region coincides approximately with the inferior part of the region with anomalous diffusion. We observe that Thule is on the border of this region and its orbit shows a typical behavior of anomalous diffusion with $H<1.55$.

Acknowledgements. The authors would like to thank Dr. Sylvio Ferraz-Mello for several useful comments. This work was supported by the Conselho Nacional de Desenvolvimentos Científico e Tecnológico (CNPq-The Brazilian National Council of Scientific and Technologic Developments) and by the Fundação de Amparo a Pesquisa do Estado de Minas Gerais (FAPEMIG-The Research Fostering Foundation of the State of Minas Gerais).

\section{References}

Addison, P. 1997, Fractals and Chaos (New York: Institute of Physics Pub.), 54

Berg, H. C. 1983. Random Walk in Biology (Princeton: Princeton Univ. Press.), 9

Chandrasekhar, S. 1941, ApJ, 94, 512

Chandrasekhar, S. 1943, Rev. Mod. Phys., 15, 1

Chirikov, B. V. 1979, Phys. Rep., 52, 263

Cordeiro, R. R., Gomes, R. S., \& Vieira Martins, R. 1997, Celes. Mech. Dyn. Astron., 65, 407

Efthymiopoulos, C., Contopoulos, G., Voglis, N., \& Dvorak, R. 1997, J. Phys. A: Math. Gen., 30, 8167

Everhart, E. 1985, in Dynamics of Comets: Their Origin and Evolution, ed. A. Carusi, \& G. B. Valsechi (Dordrecht: Reidel), 185

Feder, J. 1988, Fractal (New York: Plenum Press), 163

Kampen, K. G. van, 1982, Stochastic Process in Physics and Chemistry (Amsterdam: North-Holland), 209
Kandrup, H. E., Siopis, C., Contopoulos, G., \& Dvorak, R. 1999, Chaos, 9-2, 381

Klafke, J. 2001, Estudo da Difusão Caótica em Ressonâncias Asteroidais, Doctoral tesis, Universidade de São Paulo, São Paulo, 73

Klafter, J., Shlesinger, M. F., \& Zumofen, G. 1996, Phy. Today, February, 33

Laskar, J. 1990, Icarus, 88, 266

Mandelbrot, B. B. 1982, The Fractal Geometry of Nature (New York: W. H. Freeman), 247

Mandelbrot, B. B., \& Van Ness, J. W. 1968, SIAM Rev., 10, 422

Metzler, R., \& Klafter, J. 2000, Phy. Rep., 339, 1

Milani, A., \& Nobili, A. M. 1997, AJ, 114, 1246

Morbidelli, A., Zappala, V., Moons, M., Cellino, A., \& Gonzi, R. 1995, Icarus, 118, 132

Murray, N., \& Holman, M. 1997, AJ, 114, 1246

Murray, N., \& Holman, M. 1999, Science, 283, 1877

Nesvorný, D., \& Ferraz-Mello, S. 1997, Icarus, 130, 247

Paul, W., \& Baschnagel, J. 1999, Stochastic processes: From Physics to Finance (Berlin: Springer), 5

Robutel, P., \& Laskar, J. 1990, Icarus, 152, 4

Roig, F., Nesvorný, D., \& Ferraz-Mello, S. 2002, MnRAS, 335, 417

Shlesinger, M. F., Zaslavsky, M., \& Klafter, J. 1993, Nature, 363, 31

Smith, T. B. 2001, Icarus, 151, 123

Tsiganis, K., Varvoglis, H., \& Hadjidemetriou, J. D. 2000, Icarus, 146, 240

Uhlenbeck, G. E., \& Ornstein, L. S. 1930, Phys. Rev., 36, 823

Varvoglis, H., \& Anastasiadis, A. 1996, AJ, 111, 1718

Wang, M. C., \& Uhlenbeck, G. E. 1945, Rev. Mod. Phys., 17, 323

Wisdom, J., \& Holman, M. 1992, AJ, 104, 2022

Zaslavsky, G. M. 2002, Chaos, Phy. Rep., 371, 461 